\title{
Thérapie cellulaire par cellules souches mésenchymateuses d'une atteinte multi-organes induite par une irradiation gamma : un modèle expérimental*
}

S. FRANÇOIS ${ }^{1}$, M. MOUISEDDINE ${ }^{1}$, A. SEMONT ${ }^{1}$, J. FRICK ${ }^{1}$, A. SACHÉ ${ }^{1}$, D. THIERRY ${ }^{1}$, P. VOISIN ${ }^{1}$, N.-C. GORIN ${ }^{2,3}$, P. GOURMELON ${ }^{1}$, A. CHAPEL ${ }^{1}$

(Manuscrit reçu le 21 décembre 2006, accepté le 17 mai 2007)

RÉSUMÉ Les cellules souches mésenchymateuses (CSM) sont présentes dans divers tissus de l'organisme adulte dont la moelle osseuse. In vitro, les CSM peuvent se différencier en de nombreux types cellulaires du mésoderme, de l'endoderme et de l'ectoderme ; in vivo, elles ont la capacité de migrer vers un organe lésé. Il existe cependant peu d'informations sur le devenir et le potentiel thérapeutique lors de l'injection de ces cellules souches dans un organisme ayant subit une irradiation accidentelle ou thérapeutique. Nos travaux ont permis de mettre en évidence la répartition spatiale et le taux de prise de greffe des CSM injectées par voie intraveineuse (IV) dans un organisme en fonction de la configuration pour une irradiation gamma. Les CSM ont été isolées à partir de moelle osseuse humaine (CSMh) et injectées à des souris immunodéficientes $\mathbf{2 4}$ heures après irradiation. Nous avons mis au point trois types de configurations, une configuration d'irradiation corps entier (ICE) à une dose sublétale de 3,5 Gy et deux configurations d'irradiations localisées, pour lesquelles les souris reçoivent une dose locale totale de $8 \mathrm{~Gy}$ au niveau de l'abdomen ou de 30 Gy au niveau de la patte droite postérieure. Le taux d'implantation des CSMh dans les différents organes de l'organisme 15 jours après irradiation a été quantifié par amplification du gène humain de la bêta-Globine par PCR. Puis leur localisation in situ a été mise en évidence par marquage immuno-histochimique de la bêta-2microglobuline humaine sur des sections d'organes murins possédant de l'ADN humain. En absence d'irradiation, les CSMh ont été détectées en très faible quantité. En revanche, le taux de CSMh implantées est plus important au niveau des zones irradiées à forte dose, suggérant que la colonisation des CSMh dans les tissus après irradiation est dépendante de la configuration d'irradiation. L'implantation des CSMh dans les organes n'appartenant pas aux zones surexposées évoque un état inflammatoire généralisé radio-induit. Cette observation met en avant l'existence d'un effet à distance (abscopal) des atteintes tissulaires locales radio-induites. L'ensemble de nos résultats suggère que la thérapie cellulaire par les CSM pourrait

* Ce travail a été soutenu par des financements EDF (Comité de radioprotection) et CEE 6 e PCRD (contrat FIRST numéro 503436). Sabine François a bénéficié d'une bourse d'étude de la région Ile-de-France. Cette étude a bénéficié du soutien du comité de radioprotection d'EDF et de la région Ile-de-France. Nous remercions Patrice Richard et Magali Leroy pour leur précieuse contribution.

IRSN, Direction de la radioprotection de l'Homme, B.P. 17, 92262 Fontenay-aux-Roses Cedex, France.

2 Laboratoire de Thérapie Cellulaire et Radioprotection Accidentelle, EA 1638, Faculté de Médecine Saint-Antoine, Université Paris VI Pierre et Marie Curie, 27 rue de Chaligny, 75012 Paris, France.

3 Service d'Hématologie et de Thérapie cellulaire, Hôpital Saint-Antoine, 75012 Paris, France. 
être utilisée pour la régénération des tissus normaux lésés suite à une irradiation accidentelle ou chez les patients soumis à une radiothérapie.

ABSTRACT Cellular therapy by mesenchymal stem cells in the gamma radiation-induced multiorgan dysfunction syndrome.

Mesenchymal stem cells (MSCs) have been shown to migrate to various tissues. There is little information on the fate and potential therapeutic efficacy of the reinfusion of MSCs following total body irradiation (TBI). We addressed this question using human MSC (hMSCs) infused to nonobese diabetic/severe combined immunodeficient (NOD/SCID) mice submitted to TBI. Further, we tested the impact of additional local irradiation (ALI) superimposed to TBI, as a model of accidental irradiation. NOD/SCID mice were transplanted with hMSCs. Group 1 was not irradiated before receiving hMSC infusion. Group 2 received only TBI at a dose of $3.5 \mathrm{~Gy}$, group 3 received local irradiation to the abdomen at a dose of $4.5 \mathrm{~Gy}$ in addition to TBI, and group 4 received local irradiation to the leg at $26.5 \mathrm{~Gy}$ in addition to TBI. Fifteen days after gamma irradiation, quantitative and spatia distribution of the hMSCs were studied. Histological analysis of mouse tissues confirmed the presence of radio-induced lesions in the irradiated fields. Following their infusion into nonirradiated animals, hMSCs homed at a very low level to various tissues (lung, bone marrow, and muscles) and no significant engraftment was found in other organs. TBI induced an increase of engraftment levels of hMSCs in the brain, heart, bone marrow, and muscles. Abdominal irradiation (AI) as compared with leg irradiation (LI) increased hMSC engraftment in the exposed area (the gut, liver, and spleen). Comparison of two local irradiations has shown that (LI) as compared with (AI) increased hMSC engraftment in the exposed area. An increase of hMSC engraftment in organs outside the fields of the ALI was also observed. Conversely, following LI, hMSC engraftment was increased in the brain as compared with AI. This study shows that engraftment of hMSCs in NOD/ SCID mice with significantly increased in response to tissue injuries following TBI with or without ALI. ALI induced an increase of the level of engraftment at sites outside the local irradiation field, thus suggesting a distant (abscopal) effect of radiation damage. This work supports the use of MSCs to repair damaged normal tissues following accidental irradiation and possibly in patients submitted to radiotherapy.

Keywords: mesenchymal stem cell / injured tissues / homing / transplantation / irradiation

\section{Abreviations}

CSM : Cellules Souches Mésenchymateuses; CSMh : CSM humaines; ICE : Irradiation Corps Entier ; IA : Irradiation Abdominale ; IP : Irradiation de la patte droite postérieure ; IV : Intraveineuse ; Gy : Gray.

\section{Introduction}

La prise en charge d'une personne irradiée accidentellement est complexe. Celleci dépend principalement de la dose et de la géométrie d'irradiation. Plusieurs syndromes radio-induits peuvent se développer simultanément en fonction de la dose reçue : le syndrome hématopoḯtique (Bertho et al., 2005), le syndrome gastro-intestinal (Monti et al., 2005) et le syndrome cérébro-vasculaire (Gourmelon et al., 2005). Dans un contexte d'inflammation généralisée et de 
réactions immunologiques ces phénomènes regroupés peuvent se combiner pour former le syndrome d'atteinte multi-organes radio-induit (ou Multiple Organ Dysfunction Syndrome : MODS) (Hirama et al., 2003). Tandis que les traitements du syndrome hématopoïétique (facteurs de croissance) ont démontré une certaine efficacité, la prise en charge thérapeutique de lésions tissulaires autres telle que celle du tractus digestif, de la peau et les poumons reste délicate. À ce jour, la diminution des cellules progénitrices capables de régénérer les tissus semble être l'une des principales causes de ces dysfonctionnements. En apportant des cellules souches exogènes, la thérapie cellulaire pourrait être une stratégie innovante pour le traitement des irradiations accidentelles, une fois que l'absence d'effet secondaire aura été écartée. Notre étude se place dans l'évaluation d'une approche thérapeutique du syndrome multi-organes par un apport de cellules souches mésenchymateuses (CSM) qui possèdent un potentiel multiple de différenciation en cellules des lignées ostéocytaires, chondrocytaires, adipocytaires, et en cellules stromales (Deans et Moseley, 2000 ; Pittenger et al., 1999 ; Mouiseddine et al., 2007).

Nous avons recherché si les CSM peuvent remplacer les cellules progénitrices dans les organes irradiés. Cette substitution limiterait les atteintes radio-induites et accélérerait la régénération tissulaire. Pour répondre à cette question, l'implantation des CSM humaines dans les territoires irradiés a été explorée. Afin de quantifier la colonisation des CSM humaines dans les organes irradiés, des configurations d'irradiations corps entier et locales ont été développées chez la souris. Dans cette étude, les cellules humaines ont été injectées au modèle de souris immunodéficientes (NOD/SCID). Ceci permettant la tolérance des CSM humaines chez les animaux receveurs.

Nos résultats ont montré que les $\mathrm{CSMh}$, après injection à des souris adultes non irradiées, pouvaient migrer dans la moelle osseuse et les muscles. L'irradiation corps entier (ICE) a entrâné une augmentation de la prise de greffe des CSMh dans la moelle osseuse, les muscles, et dans d'autres organes tels que le cerveau, le cœur, et le foie en comparaison aux animaux non irradiés. Lors des irradiations localisées, les CSMh s'implantent préférentiellement dans les zones surexposées. De plus l'implantation des CSMh dans les organes n'appartenant pas aux zones surexposées évoquant un état inflammatoire généralisé radio-induit, soulignant l'existence d'un effet à distance (abscopal) des organes appartenant aux zones irradiées sur les tissus en dehors du champ d'irradiation. Cet effet abscopal semble être le résultat de la libération de médiateurs inflammatoires radio-induits (Snyder, 2004 ; Van der Meeren et al., 2005). Ces résultats suggèrent que la thérapie cellulaire par les CSM pourrait être utilisée pour la régénération des tissus normaux lésés suite à une irradiation. 


\section{Matériel et méthodes}

\subsection{Isolation, purification et expansion des CSM humaines (CSMh)}

Les cellules souches mésenchymateuses ont été obtenues à partir d'échantillons de moelle osseuse prélevés au niveau de la crête iliaque de sujets volontaires sains. Ces prélèvements de moelle osseuse (MO) ont été réalisés sous anti-coagulant (héparine de lithium; Choay ${ }^{\circledR}$ Sanofi-Synthélabo) et les cellules souches mésenchymateuses ont été cultivées selon le protocole décrit précédemment (François et al., 2006b). Le phénotype et le pouvoir de différenciation des CSM isolées de moelle osseuse en cellules des lignées ostéoblastiques, chondrocytaires, adipocytaires ont été étudiés selon les protocoles décrits précédemment (Mouiseddine et al., 2007).

\subsection{Configuration d'irradiation et colonisation des CSMh dans les tissus irradiés}

Toutes les expérimentations et procédures ont été réalisées conformément à la réglementation du Ministère de l'agriculture français sur l'expérimentation animale (loi $\mathrm{n}^{\circ}$ 87-847 du 19 octobre 1987, modifiée en mai 2001). Les souris NOD-LtSz-scid/scid (NOD-SCID) qui ont été utilisées proviennent de couples reproducteurs achetés auprès du laboratoire Jackson (Bar Harbor, Maine, ÉtatsUnis). Les animaux ont été élevés dans notre laboratoire dans des cages de microisolement stérile. Les irradiations ont été réalisées avec une source de ${ }^{137} \mathrm{Cs}$ (IBL 637 Cis Bio international), avec un débit de dose $0,38 \mathrm{~Gy} / \mathrm{mn}$. Vingt quatre heures après exposition aux rayonnements ionisants, les CSMh ont été injectées par voie intraveineuse à une concentration de $5 \times 10^{6} \mathrm{CSMh}$. Cette étude a été réalisée avec 5 groupes d'animaux chacun constitué de 10 souris NOD/SCID agées de 8 semaines. Le groupe 1 représente les souris non irradiées recevant une injection de CSMh. Les groupes 2, 3 et 4 ont tous reçu une irradiation corps entier (ICE) à une dose sublétale de 3,5 Gray, avant injection. Les groupes 3 et 4 ont reçu respectivement une dose supplémentaire locale d'irradiation au niveau de l'abdomen (IA) de 4,5 Gy ou au niveau de la patte droite postérieure (IP) de 26,5 Gy. Les animaux ont été sacrifiés 15 jours post irradiation. Le sang périphérique, la moelle osseuse des fémurs, le cœur, les poumons, le foie, les reins, la rate, l'estomac, l'intestin, le cerveau ainsi que les quadriceps, les tibias, et la peau de la patte postérieure droite ont été prélevés. La prise de greffe des CSMh dans les différents tissus a été évaluée, par quantification du gène de la bêtaglobine humaine, à 15 jours post-irradiation selon les 3 configurations d'irradiation ainsi que dans les tissus non surexposés des souris. Sur les organes dans lesquels la présence d'ADN humain était mesurable, nous avons ensuite mis 
en évidence les cellules humaines implantées par immunomarquage de la protéine bêta-2-microglobuline humaine. La dilution de l'anticorps primaire ainsi que le démasquage antigénique varient suivant le type tissulaire (Semont et al., 2006 ; Francois, 2006).

\subsection{Analyse statistique}

Les analyses statistiques ont été réalisées à l'aide du logiciel Sigmastat (SPSS, Paris, France). Les résultats sont présentés sous forme de moyenne \pm écart standard à la moyenne (ESM). Les différents groupes ont été comparés par le test-t de Student. Les différences ont été considérées comme significatives pour une valeur de $\mathrm{p}<0,05(*), \mathrm{p}<0,01(* *)$ et $\mathrm{p}<0,001(* * *)$, la non significativité a été notée (ns).

\section{Résultats}

\subsection{Isolement et caractérisation des CSMh isolées à partir de moelle osseuse}

L'analyse phénotypique illustrée par la figure 1 a permis de déterminer que les CSMh en P2 utilisées au cours de cette étude sont fortement positives pour les antigènes de surface spécifiques des CSM tels que CD105 (SH2 : 37,3\% \% 4,0) et CD73 (SH3 : 72,9\% $\pm 3,7$ ). La contamination en cellules hématopoïétiques dans nos greffons peut être considérée comme quasiment nulle $(0,2 \% \pm 0,1$ de cellules CD 45+). Le facteur d'amplification des cellules mononuclées a été estimé de 3443 à 20663 pour les différents échantillons de moelle osseuse utilisés pour la réalisation de nos greffons.

\subsection{L'exposition aux rayonnements ionisants induit des lésions tissulaires chez les souris contrôles non greffées : analyse histologique}

Quinze jours après une ICE à 3,5 Gy, une déplétion cellulaire a été observée au niveau de la rate (Fig. 2A) et des hémorragies au sein de la moelle osseuse (Fig. 2B). Après une irradiation abdominale de $8 \mathrm{~Gy}$ une atrophie des villosités, une désorganisation des cryptes, et une infiltration inflammatoire sous la muqueuse ont été observées au niveau de l'intestin (Fig. 2C, flèche verte indiquant l'inflammation). L'irradiation locale de la patte postérieure a induit au niveau de la peau des zones d'ulcération et une modification de l'épaisseur du derme (Fig. 2D, flèche noire indiquant une ulcération). Aucune lésion n'a été observée dans les tissus non irradiés (Figs. 2E, 2F, 2G, et 2H). 


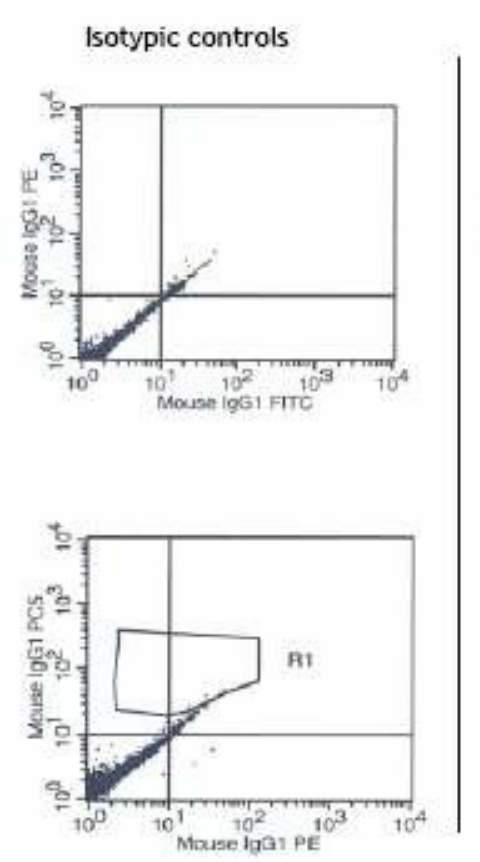

\section{CD73/CD105/CD45 Staining}
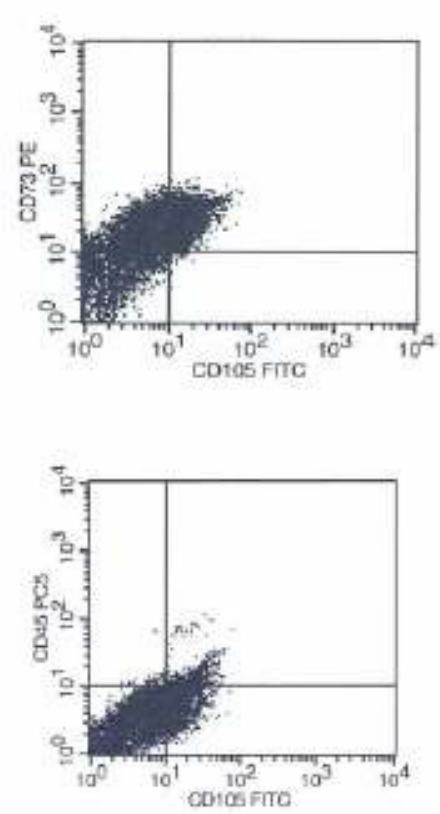

Figure 1 - Analyse par cytométrie en flux d'un échantillon de CSMh en P2. Représentation graphique de l'analyse de cytométrie en flux d'un échantillon de CSMh en P2 : détermination du taux de cellules possédant les marqueurs antigéniques de surfaces spécifiques des CSM, SH2 (CD105) et SH3 (CD73) et du taux de cellules possédant le marqueur CD45 (marqueur des cellules hématopoïétiques).

Determination of frequency of positive cells for specific markers of hMSC at second passage, SH2 (CD105) and SH3 (CD73) and positive cells for CD45 (haematopoietic cell marker), representative FACS plot analysis of the graft sample.

\subsection{Les CSMh injectées sont détectées en très faible quantité dans les tissus des souris NOD/SCID non irradiées}

Les résultats de l'analyse par PCR quantitative ont permis de mettre en évidence que les CSMh injectées à des animaux non irradiés $(n=10)$, pouvaient coloniser en faible quantité de nombreux tissus. Quatorze jours après leur injection, les CSMh sont détectées dans les poumons à un taux de $0,06 \%$ (Fig. 3B), et de $0,14 \%$ dans la moelle osseuse (Fig. 5D). Aucune prise de greffe significative n'a été constatée dans le cerveau (Fig. 3A), les reins (Fig. 4B), le foie (Fig. 4A), l'intestin (Fig. 4E), l'estomac, le cœur (Fig. 4C), la peau (Fig. 5A), les muscles (Fig. 5B), les os (Fig. 5C), et le sang périphérique. 


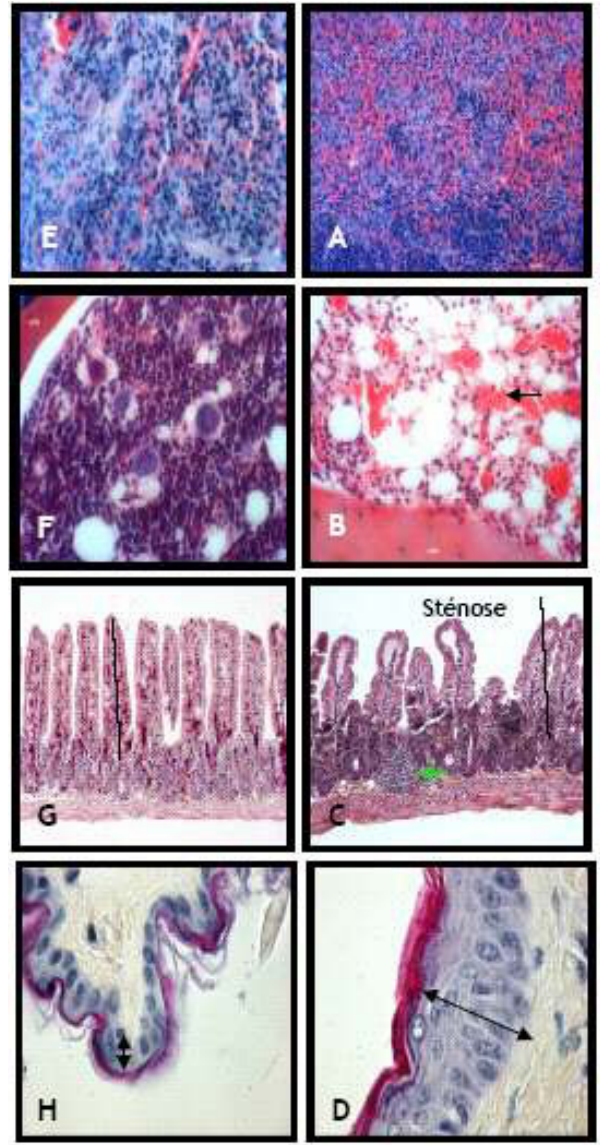

Figure 2-Analyse histologique des tissus après irradiation. Analyse histologique des tissus prélevés par un marquage HES (Hemalin, Eosine, Safran): (A) déplétion cellulaire au niveau de la rate après irradiation ; (B) moelle osseuse hémorragique (flèche noire); $(C)$ intestin de souris après IA observation d'atrophie des villosités et d'infiltrats inflammatoires (flèche verte) au niveau de la sous muqueuse ; (D) observation de modification de l'épaisseur du derme de la peau des patte irradiées ; $(E)$ rate contrôle ; (F) moelle osseuse contrôle ; (G) intestin contrôle ; (H) peau contrôle.

Histological examination of radiation exposed tissues by HES staining: (A) spleen with cellular depletion; (B) bone marrow with haemorrhage (black arrow); (C) gut with villus atrophy and inflammatory infiltrates of the sub mucosal layer (green arrow) and (D) modification of the thickness of dermis of irradiated right hind leg (black arrow). Non-irradiated controls: spleen $(E)$, bone marrow $(F)$, gut $(G), \operatorname{skin}(H)$.

\subsection{L'ICE favorise la prise de greffe des CSMh dans un plus grand nombre d'organes}

Des taux d'implantation plus élevés des CSMh ont été observés après une ICE dans le cerveau $(0,07 \%$, Fig. 3A), le cœur $(0,05 \%$, Fig. 3C), le foie $(0,11 \%$, Fig. 4A), la moelle osseuse (0,37\%, Fig. 5D), et les muscles $(0,12 \%$, Fig. $5 \mathrm{~B})$. L'ICE a induit une augmentation significative de la prise de greffe des CSMh d'un facteur 2,8 dans le cerveau ( $p<0,05)$, d'un facteur 3 dans le coeur ( $p<0,05)$, d'un facteur 2,5 dans le foie $(p<0,05)$, d'un facteur 2,6 dans la moelle osseuse ( $\mathrm{p}<0,001)$, et d'un facteur 1,7 dans les muscles $(\mathrm{p}<0,05)$, en comparaison aux souris greffées non irradiées. En revanche, l'ICE n'a pas entraîné de variation 

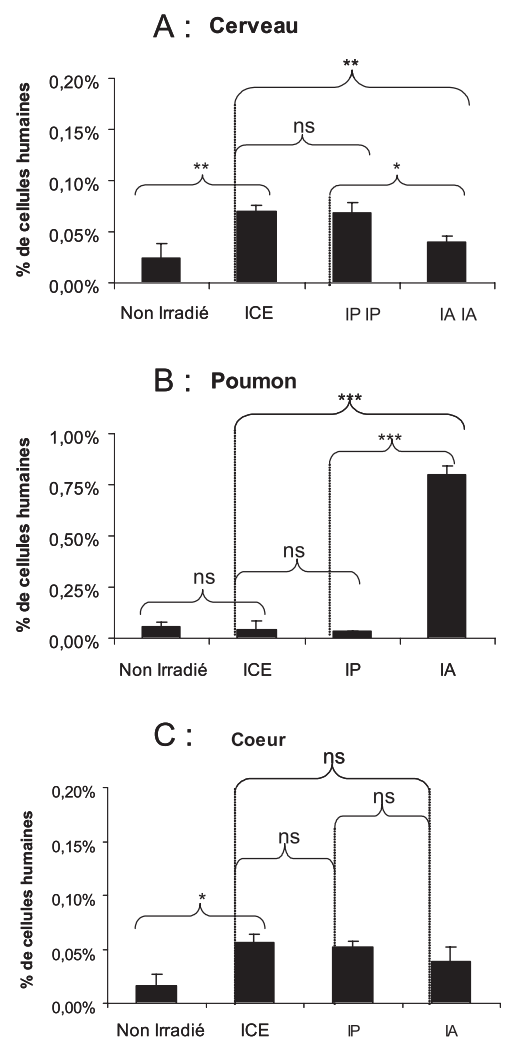

Figure 3 - Pourcentage d'implantation des CSMh. Comparaison des taux d'implantation des CSMh dans le cerveau, les poumons et le cour de souris selon les 4 conditions expérimentales (non-irradié, ICE, IP et IA) : le taux de prise de greffe des CSMh a été significativement augmenté dans le cerveau après irradiation en comparaison au nonirradié (A). L'IA a significativement augmenté la prise de greffe des CSMh dans les poumons en comparaison des autres conditions expérimentales (non-irradié, ICE et IP) (B). Le taux d'implantation des CSMh dans le cour a été augmenté par rapport aux souris non irradiées et est le même dans les 3 configurations d'irradiation (ICE, IP et IA) (C). Les résultats sont représentés sous forme de moyenne \pm écart standard à la moyenne. Les différences sont considérés significatives pour une valeur de $p<0,05(*), p<0,01$ (**) et p <0,001 (***), la non-significativité a été notée (ns).

Comparison of implantations of hMSC in brain, lung and heart tissues for mice irradiated according to three configurations of irradiation (non irradiated control mice, TBI, right posterior leg exposure or IA). hMSC engraftment rate was significantly increased in brain following irradiation, when compared to controls (A). Abdominal irradiation significantly increased hMSC engraftment in lung when compared to other settings $(B)$. hMSC engraftment in heart tissues was significantly higher following irradiation of all type than in non-irradiated controls $(C)$. All values were expressed as the mean and SEM (standard error of the mean). Significance for all analysis was set at $p<0.05(*), p<0.01(* * *), p<0.0011^{* * *)}$ and non significance was noted by (ns). 

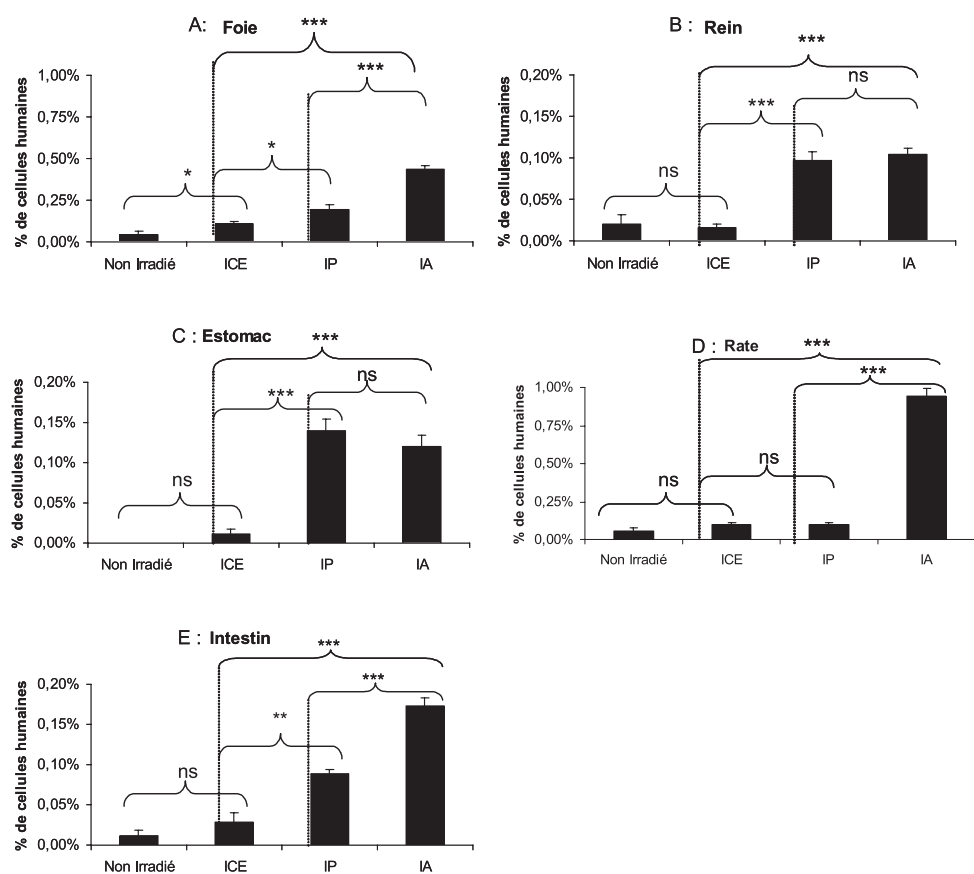

Figures 4 - Pourcentage d'implantation des CSMh. Comparaison des taux d'implantation des CSMh dans les organes appartenant à la sphère abdominale des souris selon les 4 conditions expérimentales (non-irradié, ICE, IP et IA). Le taux d'implantation des CSMh a été significativement augmenté dans le foie quelle que soit la configuration d'irradiation, en comparaison aux souris nonirradiées (A). La prise de greffe des CSMh a été augmentée dans les reins et l'estomac dans les deux conditions expérimentales d'irradiation localisée en comparaison à l'ICE et aux souris non irradiées (B et C). Une augmentation significative du taux d'implantation des CSM h dans la rate a été observée après une IA en comparaison aux trois autres conditions expérimentales (non irradiée, ICE et IP) (D). Les CSMh se sont significativement pas implantées dans l'intestin des souris irradiées localement en comparaison aux souris non irradiées et aux souris recevant uniquement une ICE. Les résultats sont représentés sous forme de moyenne \pm écart standard à la moyenne. Les différences sont considérés significatives pour une valeur de $p<0,05$ (*), $p<0,01$ (**) et p $<0,001$ (***), la non-significativité a été notée (ns).

Comparison of implantations of hMSC in abdominal tissues for mice irradiated according to three configurations of irradiation (TBI, IA, and right posterior leg exposure) or non-irradiated control mice. HMSC engraftment rate was significantly increased in liver when compared to controls (A). Significantly increased hMSC engraftment was observed in kidney or stomach following both local irradiations, when compared to TBI or non-irradiated controls $(B$ and $C)$. Significantly increased hMSC engraftment was observed in spleen following abdominal irradiation when compared to TBI alone, right hind leg exposure or non-irradiated controls (D). Significantly increased hMSC engraftment was observed in gut following right leg exposure or abdominal irradiation when compared to TBI or non irradiated control, $(E)$. All values were expressed as the mean and SEM (standard error of the mean).Significance for all analysis was set at $p<0.05(*), p<0.01(* * *), p<0.001$ (***) and non significance was noted by (ns). Each group was constituted of 10 animals $(n=10)$. 

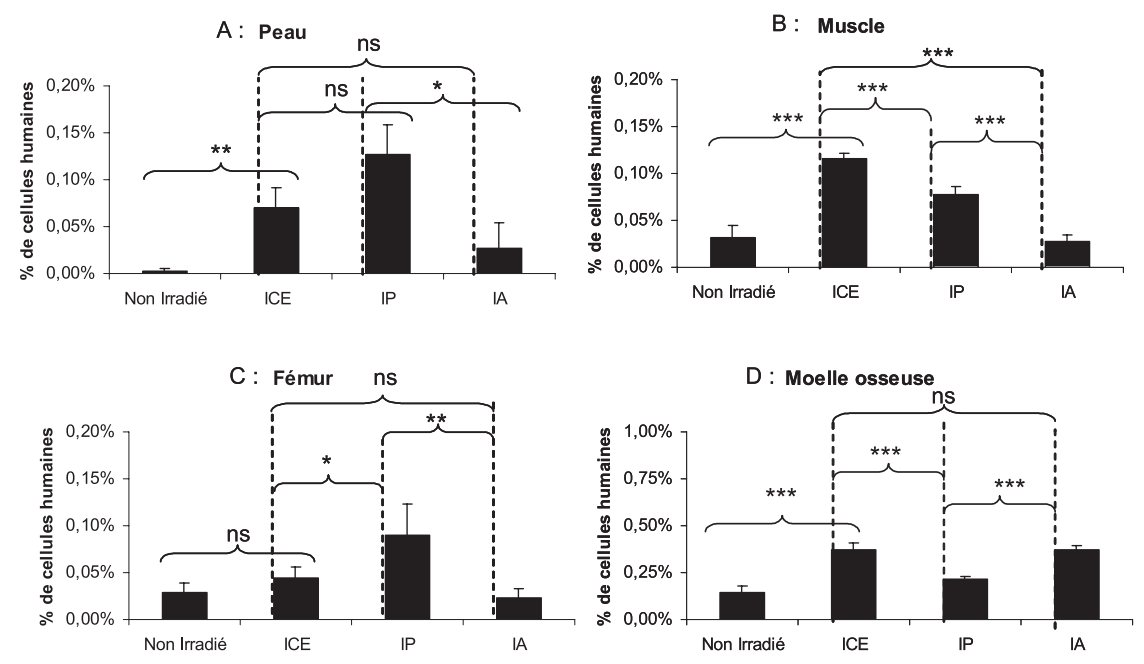

Figure 5 - Pourcentage d'implantation des CSMh Comparaison des taux d'implantation des CSMh dans les tissus appartenant à la patte postérieure droite des souris selon les 4 conditions expérimentales (non-irradié, ICE, IP et IA). Le taux d'implantation des CSMh a été significativement augmenté dans la peau $(A)$ ou dans le muscle $(B)$ de la patte droite postérieure des souris ayant reçu une ICE ou une IP par rapport aux souris non irradiées ou ayant reçu une IA. La prise de greffe des CSMh dans l'os (fémur) est significativement augmentée après une IP en comparaison aux trois autres conditions expérimentales $(C)$. Le taux d'implantation des CSMh a été augmenté dans la moelle osseuse après une ICE ou une IA en comparaison aux souris non irradiées ou ayant reçu une IP $(D)$. Les résultats sont représentés sous forme de moyenne \pm écart standard à la moyenne. Les différences sont considérés significatives pour une valeur de $p<0,05(*), p<0,01(* *)$ et $p<0,001(* * *)$, la non-significativité a été notée (ns).

Comparison of implantation of hMSC in right posterior leg tissues for mice irradiated according to three configurations of irradiation (TBI, IA, right posterior leg exposure) or nonirradiated control mice. HMSC engraftment rate was significantly increased in skin (A) or muscle (B) following TBI or right leg exposure when compared to IA or non-irradiated controls. HMSC engraftment rate in bone was significantly increased following posterior leg exposure when compared to other irradiation protocols or non-irradiated controls $(C)$. HMSC engraftment rate was significantly increased in the bone marrow following TBI or abdominal irradiation when compared to right hind leg exposure or non-irradiated controls $(D)$. All values were expressed as the mean and SEM (standard error of the mean). Significance for all analysis was set at $p<0.05(*), p<0.01(* * *), p<0.001(* * *)$, non significance (ns). Each group was constituted of 10 animals $(n=10)$.

significative de la prise de greffe des CSMh dans les poumons (0,04\%, Fig. 3B). La détection du gène de la bêta-globine humaine dans les poumons et le cœur, suggère que les CSMh circulent dans l'organisme via les vaisseaux sanguins et ce au moins jusqu'à 14 jours après leur injection. 


\subsection{Les CSMh colonisent préférentiellement les organes appartenant à la zone corporelle ayant reçu la dose d'irradiation la plus élevée}

La figure 4 illustre les taux de prise de greffe des CSMh dans les organes appartenant à la sphère abdominale. La figure 5 représente les taux de prise de greffe des CSMh dans les organes de la patte postérieure (peau, muscle, fémur et moelle osseuse). Les souris ont reçu préalablement une ICE à 3,5 Gy puis localement une irradiation au niveau de la sphère abdominale (IA) ou au niveau de la patte droite postérieure (IP). En comparaison à l'IP, l'IA a entraîné une augmentation du taux de prise de greffe des CSMh dans la zone surexposée d'un facteur 2 dans l'intestin ( $\mathrm{p}<0,001$ ) (Fig. 4E), d'un facteur 2,3 dans le foie, et d'un facteur 9,3 dans la rate (respectivement Figs. 4A et 4D). Dans les organes n'appartenant pas à l'abdomen, la prise de greffe des CSMh a été également augmentée d'un facteur 1,8 dans la moelle osseuse (Fig. 5D) et d'un facteur 11,6 dans les poumons (Fig. 3B) $(\mathrm{p}<0,05)$. À l'inverse, en comparaison à l'IA, l'IP a entraîné une augmentation significative de l'implantation des CSMh au niveau des organes appartenant à la patte irradiée d'un facteur 5 dans la peau $(\mathrm{p}<0,05 *)$, et d'un facteur 3 dans les quadriceps $\left(p<0,001^{* * *}\right)$ (Figs. 5A et 5B). La comparaison des taux de prise des CSMh entre les deux configurations d'irradiations localisées met en évidence que dans certains tissus la colonisation n'est pas dépendante de la surexposition comme l'estomac (Fig. 4C), les reins (Fig. 4B), et le tissu cardiaque (Fig. 3C). Des taux de colonisation des CSMh importants ont été observés, en particulier dans le cerveau (Fig. 3A), à la suite d'une irradiation de $30 \mathrm{~Gy}$ au niveau de la patte. L'IP, en comparaison à l'IA, a entrainé une augmentation significative de la prise de greffe des CSMh dans le cerveau d'un facteur $2,2\left(\mathrm{p}<0,05^{*}\right)$, suggérant un effet à distance (abscopal) des lésions radio-induites.

\subsection{Localisation in situ des CSMh dans les tissus irradiés par immuno- histologie et comparaison aux résultats de l'analyse PCR}

Pour localiser les cellules souches mésenchymateuses humaines au sein des tissus murins où avait été mis en évidence de l'ADN humain, nous avons procédé à une analyse histologique par immuno-marquage de l'antigène spécifique de la $\beta-2$ microglobuline humaine. Cette étude a été réalisée sur la rate de souris irradiées corps entier et sur les poumons d'animaux irradiés localement au niveau de l'abdomen. Dans la rate de souris, les cellules positives ont été localisées in situ en amas ou isolées au sein de ce tissu hématopoḯtique. À l'aide de cet immunomarquage nous avons pu quantifier la présence des cellules humaines sur la coupe de rate et observer qu'il avait $10 \%$ de cellules humaines qui recolonisaient la rate murine 15 jours après une ICE (Fig. 6A). Lors d'une IA, dans les poumons nous 

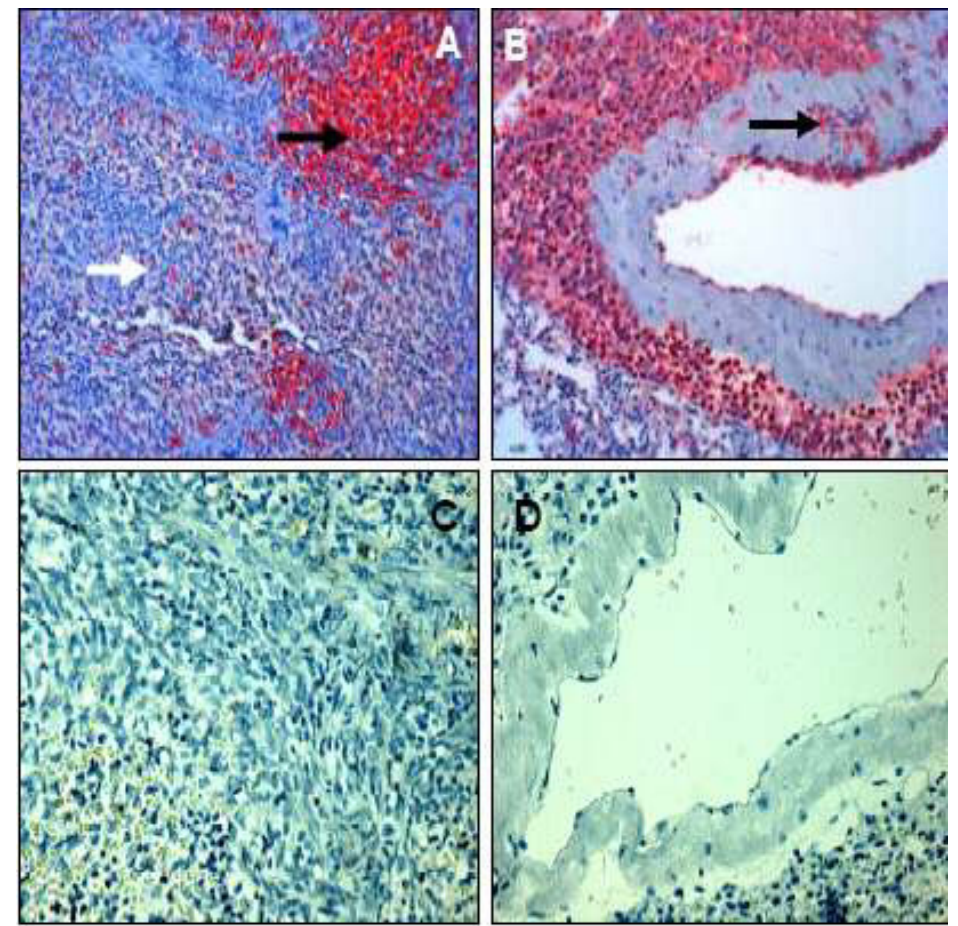

Figure 6 - Localisation in situ des CSMh dans les poumons et la rate après irradiation immuno-marquage de la bêta-2-microglobuline humaine sur coupe de rate et de poumon. Les cellules humaines exprimant la bêta-2-microglobuline sont colorées en rouge $(A)$ dans la rate 15 jours après une ICE. Les cellules sont localisées en amas (flèche blanche) ou isolées (flèche noire) au sein de l'architecture tissulaire de la rate. Dans les poumons 15 jours après une irradiation localisée (B), des amas de cellules positives pour la bêta-2-microglobuline humaine sont retrouvés autours des vaisseaux. Certaines cellules positives semblent traverser l'intima (flèche noire). Les figures $C$ et $D$ illustrent respectivement les contrôles négatifs de la rate et des poumons 15 jours après irradiation.

Human beta-2-microglobuline immunostaining in lung and spleen. The human cells expressing the human beta-2-microglobulin are stained in red; $(A)$ in spleen 15 days after TBI. Human cells were insulated (white arrow) or gathered in cluster (black arrow) in contact with conjunctive structure of spleen; $(B)$ in lung after localised radiation exposure, clusters of positive cells in perivascular position (black arrow) and passage of human cells through the vascular wall and an intravascular colonization. Figures $C$ and $D$ represents negative controls (respectively spleen and lung, 15 days after TBI).

avons observé des amas de cellules positives pour la $\beta$-2-microglobuline humaine qui ont été visualisées au niveau péri-vasculaire comme l'illustre la figure 6B. Plus précisément, nous avons pu observer que des cellules humaines semblaient traverser la paroi vasculaire. Nous avons mis en évidence une colonisation 
intra-vasculaire des CSMh sous l'intima. Les figures 6C et 6D illustrent les contrôles négatifs correspondant à la rate et aux poumons. Dans les zones périvasculaires des poumons, nous avons pu compter jusqu'à $45 \pm 2 \%$ de cellules humaines positives pour la $\beta$-2-microglobuline humaine. Néanmoins, aucune cellule humaine n'a été détectée dans les autres zones pulmonaires. En opposition à l'analyse immuno-histo-chimique, l'analyse quantitative de la prise de greffe met en évidence une prise de greffe plus faible ce qui semble normal puisque celleci prend en considération l'organe dans son ensemble. Nous n'avons pas observé de grande variabilité de la prise de greffe entre les animaux d'un même groupe. Il faut cependant noter que pour certains organes, comme le foie, la variabilité intragroupe a été légèrement plus importante.

\section{Discussion}

Nous avons étudié l'impact thérapeutique d'une greffe de CSM sur les atteintes multi organes radio-induites. Nos travaux ont permis de mettre en évidence la répartition spatiale et le taux de prise de greffe des CSM injectées par voie intraveineuse (IV) dans un organisme en fonction de la configuration d'irradiation.

Dans cette étude, nous avons établi un modèle pré-clinique dans lequel les CSMh ont été injectées à des souris NOD/SCID irradiées ou non. L'étude de l'implantation des CSM humaines, nous a imposé de travailler chez un modèle murin immunotolérant tel que la souris NOD/SCID afin de pouvoir réaliser une xénogreffe, sans avoir le souci du rejet du greffon. Nous avons préalablement validé ce modèle de greffe de cellules humaines dans un organisme murin receveur, en démontrant qu'une cellule souche humaine reste une cellule souche dans une autre espèce. Nous avions greffé des cellules souches hématopoḯtiques humaines ce qui avait permis chez la souris NOD/SCID de générer une hématopoï̀se humaine sur plusieurs semaines. De plus l'injection de CSMh a amélioré le soutien cette hématopoïèse (Bensidhoum et al., 2004). Tous les animaux irradiés ont reçu une ICE à une dose sublétale de 3,5 Gy. Pour tester l'impact des lésions entraînées par une irradiation localisée, deux groupes d'animaux ont reçu, en plus de l'irradiation corps entier, une dose au niveau de l'abdomen (8 Gy) ou au niveau de la patte postérieure droite (30 Gy).

Nous avons constaté qu'en absence d'irradiation, les CSMh pouvaient coloniser en faible quantité certains organes comme les poumons et la moelle osseuse. La colonisation des CSMh dans la moelle osseuse était attendue puisque ce tissu est leur lieu d'origine. La présence importante des CSMh au niveau des poumons pourrait être liée à l'incapacité des CSMh à traverser les capillaires pulmonaires du fait de leur grande taille, ou à se fixer aux cellules endothéliales par liaison à des récepteurs aux molécules d'adhésion (Tang et al., 2004). La mise 
en place des trois modèles d'irradiations différents a permis d'observer que l'ICE qui précède l'injection des CSMh entraîne une augmentation des taux de prise de greffe dans certains tissus, mais pas dans tous les organes des souris irradiées corps entier. Nous avons également constaté qu'à 15 jours post-irradiation (IP ou IA), les CSMh injectées par voie systémique colonisaient tous les tissus appartenant aux zones surexposées avec des taux de prise de greffe importants. Dans cette étude, nous avons détecté un taux maximal de CSMh dans la rate pour les animaux recevant une irradiation abdominale supplémentaire. Pour les animaux recevant une IP, c'est au niveau de la peau, des quadriceps et de la moelle osseuse de la patte irradiée que l'implantation des CSMh est la plus élevée.

À la suite d'une irradiation localisée, les taux de prise de greffe des CSMh ont été augmentés dans certains organes ou tissus distants de la zone surexposée. Ceci suggère une mobilisation importante des CSMh via les cytokines et chemokines vers les sites altérés. La migration vers les organes non surexposés pourrait être induite par les protéines libérées au cours de l'inflammation (Van der Meeren et al., 2004 ; Grimm et al., 2001). La détermination de ces protéines reste à se jour encore à définir clairement.

Au cours de cette étude la plupart des cellules humaines implantées ont été localisées dans des zones faiblement lésées. Des travaux précédemment menés sur un modèle de primate non humain soumis à une irradiation mixte gamma et neutronique puis injecté avec des CSM de primate non humain marquées par le gène de l'EGFP (Enhanced Green Fluorescent Protein), nous ont permis d'observer que les CSM colonisaient préférentiellement les tissus en régénération (Chapel et al., 2003). Ces résultats appuient l'hypothèse que les CSM peuvent participer à la préservation de l'intégrité cellulaire et tissulaire des tissus cibles de l'irradiation. Les mécanismes moléculaires mis en jeu pourraient se dérouler par un contact direct ou des facteurs solubles (Zhang et al., 2005).

Ces études s'inscrivent dans une démarche d'étude pré-clinique de l'utilisation de la thérapie cellulaire pour la gestion médicale des lésions précoces d'une irradiation accidentelle sévère. Nous avons montré par ailleurs que l'injection de CSM humaines diminue l'apoptose radio-induite dans l'intestin après une irradiation locale aiguë de l'abdomen (Semont et al., 2006), empêche l'apparition d'une cytotoxicité hépatique induite par une ICE (François, 2006) et favorisent la cicatrisation de la peau après une irradiation localisée (François et al., 2007). Ces études sur une irradiation uniquement localisée en absence d'irradiation corps entier montrent également des atteintes locales et une localisation similaire des CSM. Les CSM transplantées dans un organisme irradié semblent participer à la régénération tissulaire et au maintien de l'homéostasie tissulaire après irradiation (François, 2006). Ces travaux suggèrent que la thérapie cellulaire par les CSM est 
une stratégie innovante et efficace pour la régénération des tissus lésés à la suite d'une irradiation accidentelle. Il a été récemment mis en évidence que les CSM pouvaient se transformer en cellules cancéreuses in vivo. Un temps court de culture des CSM semble donc nécessaire pour obtenir des cellules transplantables sans avoir le souci d'implanter un greffon potentiellement tumorigène (Miura et al., 2006 ; Rubio et al., 2005). Bien que Rojas et al. ont montré un effet protecteur d'une injection de CSM sur la formation de la fibrose (Rojas et $\mathrm{Xu}, 2005$ ), il est évident que l'application de cette thérapeutique impose de vérifier à long terme que les CSM n'ont pas d'effets secondaires. À cet effet nous avons très récemment vérifié avant réinjection que nos CSM cultivées n'étaient pas transformées c'està-dire qu'aucune activité télomérasique n'a été détectée. En revanche, il a été publié que les CSM pourraient favoriser la croissance des cellules tumorales (Zhu et al., 2006), cependant cet effet demeure controversé (Djouad et al., 2003).

À ce jour cette stratégie thérapeutique a déjà fait ses preuves pour le traitement d'un patient ayant subi des brûlures cutanées radiologiques à la main et au dos lors d'un accident au Chili. Le patient a été traité avec succès par une thérapie innovante de greffe de peau et d'injections autologues de CSM autour de la lésion (François et al., 2006a).

L'intérêt de cette expérience, par delà son aspect spectaculaire, est d'avoir réussi à reconstituer une structure fonctionnelle composée de plusieurs tissus différents. L'ensemble de ces résultats donnant un pouvoir protecteur aux CSM implantées dans les tissus altérés, ouvrent dans un avenir proche, une alternative prometteuse à la transplantation d'organes ainsi que dans le traitement des patients soumis à une radiothérapie dans le but de limiter les atteintes aux tissus sains proches de la zone tumorale traitée.

\section{RÉFÉRENCES}

Bensidhoum M., Chapel A., François S., Mazurier C., Bouchet S., Bertho J.M., Gourmelon P., Thierry D., Lopez M. (2004) Homing of in vitro expanded STRO1- or STRO1+ human mesenchymal stem cells into the NOD/SCID mouse and their role in supporting human CD34 cell engraftment, Blood 103, 3313-3319.

Bertho J.M., Prat M., Frick J., Demarquay C., Gaugler M.H., Dudoignon N., Clairand I., Chapel A., Gorin N.C., Thierry D., Gourmelon P. (2005) Application of autologous hematopoietic cell therapy to a nonhuman primate model of heterogeneous high-dose irradiation, Radiat. Res. 163, 557-570.

Chapel A., Bertho J.M., Bensidhoum M., Fouillard L., Young R.G., Frick J., Demarquay C., Cuvelier F., Mathieu E., Trompier F., Dudoignon N., Germain C., Mazurier C., Aigueperse J., Borneman J., Gorin N.C., Gourmelon P., Thierry D. (2003) Mesenchymal stem cells home to injured tissues when co-infused with hematopoietic cells to treat a radiation-induced multi-organ failure syndrome, J. Gen. Med. 5, 1028-1038.

Deans R.J., Moseley A.B. (2000) Mesenchymal stem cells: biology and potential clinical uses, Exp. Hematol. 28, 875-884. 
Djouad F., Plence P., Bony C., Tropel P., Apparailly F., Sany J., Le Noe D., Jorgensen C. (2003) Immunosuppressive effect of mesenchymal stem cells favors tumor growth in allogeneic animals, Blood 102, 10.

François S. (2006) Étude de la capacité plastique des cellules souches mésenchymateuses humaines après irradiation du tissu receuveur: approche thérapeutique de l'atteinte multi-organe radioinduite, thèse de l'université de Versailles/Saint-Quentin-en-Yvelines.

François S., Mouiseddine M., Sache A., Mathieu N., Semont A., Voisin P., Thierry D., Chapel A. (2006a) Les cellules mésenchymateuses favorisent la cicatrisation des lésions cutanées radioinduites, Radioprotection 41, 441-454.

François S., Bensidhoum M., Mouiseddine M., Mazurier C., Allenet B., Semont A., Frick J., Saché A., Bouchet S., Thierry D., Gourmelon P., Gorin N.C., Chapel A. (2006b) Local irradiation not only induces homing of human mesenchymal stem cells at exposed sites but promotes their widespread engraftment to multiple organs: a study of their quantitative distribution after irradiation damage, Stem Cells 24, 1020-1029.

François S., Bensidhoum M., Mouiseddine M., Mazurier C., Allenet B., Semont A., Frick J., Saché A., Bouchet S., Thierry D., Gourmelon P., Gorin N.C., Chapel A. (2007) Human mesenchymal stem cells favour healing of the cutaneous radiation syndrome in a xenogenic transplant model, Ann. Hematol. 86, 1-8.

Gourmelon P., Marquette C., Agay D., Mathieu J., Clarencon D. (2005) Involvement of the central nervous system in radiation-induced multi-organ dysfunction and/or failure, BJR Suppl. 27, 6268.

Grimm P.C., Nickerson P., Jeffery J., Savani R.C., Gough J., McKenna R.M., Stern E., Rush D.N (2001) Neointimal and tubulointerstitial infiltration by recipient mesenchymal cells in chronic renal-allograft rejection, N. Engl. J. Med. 345, 93-97.

Hirama T., Tanosaki S., Kandatsu S., Kuroiwa N., Kamada T., Tsuji H., Yamada S., Katoh H., Yamamoto N., Tsujii H., Suzuki G., Akashi M. (2003) Initial medical management of patients severely irradiated in the Tokai-mura criticality accident, Br. J. Radiol. 76, 246-253.

Monti P., Wysocki J., van der Meeren A., Griffiths N.M. (2005) The contribution of radiation-induced injury to the gastrointestinal tract in the development of multi-organ dysfunction syndrome or failure, BJR Suppl. 27, 89-94.

Mouiseddine M., Francois S., Semont A., Saché A., Frick J., Thierry D., Chapel A. (2007) Human mesenchymal stem cells home specifically to radio-injured tissues in a non-obese diabetes/severe combined immunodeficiency mouse model, Br. J. Radiol. (à paraître).

Miura M.Y., Miura Y., Padilla-Nas H.M., Shi S. (2006) Accumulated chromosomal instability in murine bone marrow mesenchymal stem cells leads to malignant transformation, Stem. Cells $\mathbf{2 4}$, 1095-1103.

Pittenger M.F., Mackay A.M., Beck S.C., Jaiswal R.K., Douglas R., Mosca J.D., Moorman M.A., Simonetti D.W., Craig S., Marshak D.R. (1999) Multilineage potential of adult human mesenchymal stem cells, Science 284, 143-147.

Rojas M., Xu J. (2005) Bone marrow-derived mesenchymal stem cells in repair of the injured lung, Am. J. Respir. Cell Mol. Biol. 33, 145-152.

Rubio D., Garcia-Castro J., Martin M.C., Bernad A. (2005) Spontaneous human adult stem cell transformation, Cancer Res. 65, 3035-3039.

Semont A., François S., Mouiseddine M., Demarquay C., Frick J., François A., Thierry D., Chapel A. (2006) Mesenchymal stem cells increase self-renewal of the small intestinal epithelium and accelerate structural recovery after radiation injury, Adv. Exp. Med. Biol. 585, 19-30.

Snyder A.R. (2004) Review of radiation-induced bystander effects, Hum. Exp. Toxicol. 23, 87-89. 
Tang Y.L., Zhao Q., Zhang Y.C., Cheng L., Liu M., Shi J., Yang Y.Z., Pan C., Ge J., Phillips M.I. (2004) Autologous mesenchymal stem cell transplantation induce VEGF and neovascularization in ischemic myocardium, Regul. Pept. 117, 3-10.

Van der Meeren A., Mouthon M.A., Vandamme M., Squiban C., Aigueperse J. (2004) Combinations of cytokines promote survival of mice and limit acute radiation damage in concert with amelioration of vascular damage, Radiat. Res. 161, 549-559.

Van der Meeren A., Monti P., Vandamme M., Squiban C., Wysocki J., Griffiths N. (2005) Abdominal radiation exposure elicits inflammatory responses and abscopal effects in the lungs of mice, Radiat. Res. 163, 144-152.

Zhang Y.Z., Fouillard L., Chapel A., Bensidhoum M., Mazurier C., Nasef A., Bouchet S., Lopez M. Thierry D., Gorin N.C., Da W.M. (2005) Mesenchymal stem cells from human proximal femurs possess immunosuppressive activity, Zhonghua Yi Zue Za Zhi 39, 2780-2784.

Zhu W., Xu W., Jiang R., Qian H., Chen M., Hu J. (2006) Mesenchymal stem cells derived from bone marrow favor tumor growth in vivo, Exp. Mol. Path. 80, 267-274. 